\title{
ANÁLISE DA IMPOSIÇÃO DE PERÍODOS DE LOCK-UP SOBRE O SALDO DE DISPONIBILIDADES MANTIDO PELOS FUNDOS MULTIMERCADOS BRASILEIROS ${ }^{1}$
}

\author{
ANALYSIS OF THE EFFECT OF THE IMPOSITION OF LOCK-UP PERIODS ON THE BALANCE \\ OF CASH AND CASH EQUIVALENTS OF BRAZILIAN MULTIMARKET FUNDS
}

\author{
Dienifer Aline Clemente de Oliveira ${ }^{2}$ \\ Graduação em Ciências Contábeis Fundação Carmelitana Mário Palmério (FUCAMP) \\ Conceito Contabilidade Ltda \\ gleison.orientador@gmail.com \\ https://orcid.org/0000-0002-7532-1410
}

Gleison de Abreu Pontes

Mestre em Ciências Contábeis PPGCC/UFU

FAGEN/UFU

dienifer aco@hotmail.com

https://orcid.org/0000-0002-8421-342X

Rodrigo Fernandes Malaquias

Doutor em Administração de Empresas EAESP/FGV

FAGEN/UFU

rodrigofmalaquias@gmail.com

http://orcid.org/0000-0002-7126-1051

\section{RESUMO}

Objetivo: O objetivo geral deste trabalho é analisar se a imposição de períodos de lock-up reduz o saldo de disponibilidades mantido pelos fundos multimercados brasileiros.

Fundamento: No cenário atual, os fundos de investimento têm desempenhado papel importante na Economia brasileira, entre eles, os multimercados têm-se destacado, por apresentarem maior rentabilidade em relação aos demais. Algumas pesquisas tentam explicar as variáveis que afetam a rentabilidade, como os períodos de lock-up, que podem ter uma relação com a disponibilidade de caixa e equivalentes de caixa dos fundos.

Método: Foi selecionada uma amostra de 79 fundos multimercados que operam com lock-up, o que totalizou 4.740 observações, no período de 2011 a 2015. Os dados foram coletados por meio do

\footnotetext{
${ }^{1}$ Artigo recebido em: 20/12/2017. Revisado por pares em: 16/03/2018. Reformulado em: 18/08/2018. Recomendado para publicação, após a segunda rodada, em: 21/08/2018 por Luiz Felipe de Araújo Pontes Girão (Editor Geral). Publicado em: 31/08/2018. Organização responsável pelo periódico: UFPB

2 Endereço Praça Armindo Paranhos, 232 F, B. Boa Vista, Monte Carmelo, MG 38500-000 DOI: https://doi.org/10.22478/ufpb.2318-1001.2018v6n3.37579
} 
Banco de Dados Economática ${ }^{\circledR}$ e no site da Comissão de Valores Mobiliários (CVM). Para a análise dos dados, foi utilizada a análise de regressão para dados em painel.

Resultados: Observou-se que os períodos de lock-up são menores no Brasil, quando comparados com pesquisas em outros mercados. Os resultados também evidenciaram que não necessariamente maiores períodos de lock-up implicam em menores saldos de disponibilidades mantidos pelos fundos de investimento da amostra.

Contribuições: Considerando os argumentos presentes na literatura de que períodos de lock-up podem beneficiar o desempenho dos fundos de investimento, por meio desta pesquisa, tem-se evidências de que os períodos de lock-up não necessariamente se relacionam com menores saldos em disponibilidades, ou seja, os benefícios proporcionados por períodos de lock-up talvez sejam operacionalizados com auxílio de outras medidas.

Palavras-chave: Fundos Multimercados. Períodos de Lock-up. Disponibilidade de Caixa.

\section{ABSTRACT}

Objective: The main objective of this paper is to analyze whether the imposition of lock-up periods reduces the balance of cash held by Brazilian multimarket funds

Background: In the current scenario, investment funds have played an important role in Brazilian Economy. Among them, multimarkets have been notable for their greater profitability in relation to the others. Some researches try to explain variables that affect profitability, such as lock-up periods, which may be related to the availability of cash and cash equivalents from funds.

Method: In order to do it, we selected a sample of 79 multimarket funds that operated with lockup, an amount of 4,740 observations in the period 2011 to 2015. Data were collected through the Economática ${ }^{\circledR}$ Database and on the website of the Brazilian Securities and Exchange Commission. For the analysis of the data, we used the regression analysis for panel data.

Results: The results showed that lock-up periods are lower in Brazil, when compared with searches in other markets. The results also showed that lock-up periods do not necessarily reduce the balance of cash and cash equivalent of the funds in the sample.

Contributions: Considering the arguments available in the literature, that lock-up periods can benefit funds' performance, through this research there are evidences that lock-up periods do not necessarily are related with lower amounts of cash equivalents; in other words, the benefits from lock-up periods maybe operationalized by fund managers with the use of other internal measures.

Keywords: Multimarket Funds. Lock-up Periods. Cash Availability.

\section{INTRODUÇÃO}

Os fundos de investimento brasileiros apresentaram um crescimento significativo na última década, tendo o seu patrimônio líquido aumentado três vezes, o que representa quase $50 \%$ de toda a riqueza gerada no País (Eid \& Rochman, 2014). Entre as categorias de fundos de investimento brasileiros, têm-se os fundos multimercados, que fazem correspondência no Brasil aos hedge funds estadunidenses (Gomes \& Cresto, 2010). Ao contrário dos hedge funds, Jordão e Moura (2011) enfatizam que os fundos multimercados brasileiros não exigem que os seus gestores invistam o próprio capital no fundo e permitem o acesso de pequenos investidores às suas cotas.

No período de 2012, por exemplo, os fundos multimercados brasileiros representaram $52 \%$ do total de fundos de investimento existentes (Caldeira, Moura, Santos \& Tessari, 2014). Entre os anos de 2006 e 2018, verifica-se que o número de contas abertas na categoria dos fundos multimer- 
cados cresceu cerca de 197\% (dados de abril de 2018), de acordo com a Associação Brasileira das Entidades dos Mercados Financeiro e de Capitais (ANBIMA, 2018). Logo, a expansão dessa categoria no País pode ter ocorrido em função de alguns dos fundos multimercados apresentarem rentabilidade superior de suas cotas, quando comparados aos demais fundos de investimento brasileiros (Malaquias \& Mamede, 2015).

Para escolher o tipo de fundo que melhor se enquadra no perfil do investidor, é necessário que algumas variáveis que afetam o risco e o retorno das cotas sejam analisados (Schutt \& Caldeira, 2016). Nesse sentido, citam-se algumas variáveis potencialmente relacionadas com a rentabilidade dos fundos multimercados, tais como o tamanho do fundo, o tipo de fundo, além da cobrança de taxas de administração e de performance (Malaquias \& Eid, 2014).

Outro fator que tem sido investigado e que pode afetar o desempenho dos fundos multimercados, corresponde à imposição de períodos de lock-up que, segundo Aragon (2007), assegura que todo investimento inicial destinado para o fundo não pode ser resgatado antes de encerrar um período pré-definido. Em pesquisa com 257 fundos estadunidenses, classificados como hedge funds, no período de 1994 a 2003, o autor concluiu que períodos de lock-up afetam positivamente o caixa desses fundos, visto que as restrições impostas agregam maior rentabilidade às cotas, em razão de haver uma significativa redução de resgates durante o período estabelecido (Aragon, 2007).

No âmbito nacional, verifica-se que a imposição de períodos de lock-up passou a ser investigada na categoria dos fundos multimercados. Como exemplo, cita-se o trabalho de Pontes, Rogers e Malaquias (2015) que, a partir de uma amostra formada por 54 fundos multimercados (totalizando 3.294 observações), denominados como long and short, chegaram à conclusão que, para a amostra selecionada, a imposição de períodos de lock-up foi um fator de pouca influência sobre a rentabilidade, quando comparados aos hedge funds dos Estados Unidos, destacando-se como principal variável explicativa, a cobrança de taxas de administração sobre a rentabilidade desses fundos (Pontes et al., 2015).

Ademais, sob a perspectiva de Aragon (2007), se o disponível de caixa mantido pelos fundos multimercados aumenta em relação à imposição de períodos de lock-up, pode-se inferir que esses impedimentos fazem parte da estratégia de investimento desses fundos para obterem rentabilidade, por isso, os investidores podem esperar que esses fundos lhes proporcionem maiores retornos (Aragon, 2007). Embora haja estudos brasileiros a respeito da gestão de fundos, bem como potenciais efeitos de restrições de resgate sobre a performance dos fundos de investimento, há ainda oportunidades de pesquisa investigando os potenciais efeitos de períodos de lock-up no saldo de disponibilidades mantidos pelos fundos multimercados.

Face ao exposto, este estudo apresenta a seguinte problemática: qual o efeito dos períodos de lock-up sobre o saldo de disponibilidades mantido pelos fundos multimercados? Desse modo, o objetivo geral consiste em analisar se a imposição de períodos de lock-up reduz o saldo de disponibilidades dos fundos multimercados brasileiros, no período de 2011 a 2015.

Apesar da existência de pesquisas sobre os fundos multimercados brasileiros, Roquete, Maranho, Klotzle e Pinto (2016) afirmam que essa categoria de fundos de investimento tem sido esquecida pela literatura, desse modo, o presente estudo se justifica à medida que acrescenta novas evidências no âmbito da categoria dos fundos multimercados brasileiros, ao investigar um fator que pode auxiliar na compreensão do modo pelo qual a rentabilidade das cotas é auferida. Este fator tem ainda desdobramentos práticos para a gestão de fundos de investimento, ao evidenciar como, em média, o comportamento da alocação de recursos em disponibilidades dos fundos pode afetar a performance das cotas que possuem maiores períodos de lock-up.

Como contribuições, espera-se que este estudo forneça indícios sobre a utilização da variável períodos lock-up e o saldo de disponibilidades, em pesquisas futuras que analisem a rentabilidade das cotas de fundos multimercados. Para o mercado financeiro, este estudo pode contribuir 
para os investidores que tendem à escolha dos fundos multimercados, tenham novos subsídios que os auxiliem na escolha dos fundos que possuem restrições de lock-up, de modo que haja consonância entre os objetivos de investimento relacionados à liquidez, rentabilidade e prazos, aspecto este que corrobora Borges e Martelanc (2015).

O presente estudo está estruturado da seguinte forma: além desta introdução, na segunda seção, apresentam-se os fundamentos teóricos utilizados na análise dos resultados; na terceira seção, são apresentados os procedimentos metodológicos empregados na pesquisa; na quarta seção, são apresentados e discutidos os resultados alcançados com o presente estudo; e, na última seção, são tecidas as considerações finais.

\section{FUNDAMENTAÇÃO TEÓRICA}

Esta seção está dividida em três partes. Na primeira, é discorrido sobre o efeito dos períodos de lock-up sobre o saldo de disponibilidades, evidenciando-se alguns estudos que sustentam a hipótese testada nesta pesquisa. Como previsto na literatura, outras variáveis no setor de fundos de investimento podem afetar o desempenho das cotas, sendo, portanto, discutidas na segunda parte. Por fim, são apresentadas as características dos fundos multimercados brasileiros na última parte desta seção.

\subsection{Períodos de Lock-up e Saldo de Disponibilidades}

Segundo Aragon (2007), períodos de lock-up tratam-se do tempo pré-fixado por um fundo de investimento, em que o investidor não pode resgatar suas cotas, tornando assim, o investimento sem liquidez. Consequentemente, esses impedimentos permitem maior eficiência no gerenciamento dos ativos, pois, durante esse período, o gestor não terá a obrigação de cobrir os resgates e poderá alocar os recursos da forma que julgar mais favorável. O autor demonstrou uma diferença de 47\% ao ano, no período de 1994 a 2003, na rentabilidade dos hedge funds estadunidenses com períodos de lock-up, evidenciando que essa imposição proporcionou resultados superiores aos fundos que não operaram com a fixação do tempo de resgate. Ademais, podem ser citados outros potencias fatores relacionados a maiores saldos em disponibilidades de caixa, como saldo necessário para garantia de operações em câmbio, operações com taxas de juros ou com commodities.

Sadka (2010) afirma que quanto maior o período de lock-up de um fundo, maior será o retorno e a iliquidez das cotas, visto que um fundo que possui alto risco de liquidez e não impõe restrições de resgate, poderá sofrer grandes perdas, caso os investidores resolvam fazer o resgate de suas cotas de forma inesperada. Hong (2014) corrobora esses argumentos no contexto dos hedge funds, ao citar que o uso de períodos de lock-up permite aos gestores, além de se prevenirem de resgates inesperados, realizarem o gerenciamento do risco de liquidez (dificuldade na compra ou venda de um ativo, ao preço desejado, no tempo desejado).

Alonso (2008) contribui com a discussão, afirmando que fundos que estabelecem períodos de lock-up cobram, em caso de resgate antecipado, multas extremamente altas, o que pode ser interpretado como taxas de saídas, na visão de Aragon (2007). Nanda, Narayanan e Warther (2000) explicam que o resgate frequente das cotas pode afetar o desempenho dos fundos, visto que essa solicitação reduz o retorno médio do investimento, por conseguinte, reduz-se a rentabilidade que o gestor do fundo conseguiria alcançar.

A imposição de períodos de lock-up guarda, portanto, relação com o saldo de disponibilidades mantido pelos fundos, pois, segundo Ackermann et al. (1999), o caixa dos fundos de investimento pode sofrer variações positivas, devido às restrições de resgate, em detrimento dessa política tornar as cotas ilíquidas até o final do período acordado, faz com que ocorra a diminuição dos 
desembolsos e, consequentemente, proporcione rentabilidade superior para os cotistas (Aragon, 2007; Agarwal, Daniel \& Naik, 2009).

Dessa forma, fundos que operam com a imposição de períodos de lock-up podem proporcionar aos gestores maior liberdade para gerenciar sua carteira de ativos, já que não precisam preocupar-se com a venda de seus ativos em momentos inoportunos, para atender o resgate de suas cotas (Agarwal et al., 2009). Por conseguinte, maiores saldos de disponibilidades seriam obtidos por esses fundos, e serem utilizados como garantia em outras operações.

Aragon (2007) esclarece que os custos de transação são menores para as cotas mais ilíquidas, visto que esses custos podem ser amortizados em períodos maiores e, assim, ter minimizado o seu impacto sobre o valor das cotas. Para Fonseca e Malaquias (2012), o aumento nos resgates das cotas afeta a performance dos fundos multimercados, já que os fundos teriam que levantar recursos de caixa rapidamente para cobrir essas retiradas, assim, é importante que se tenham períodos de lock-up para que as saídas de caixa sejam reduzidas e aumente-se a rentabilidade das cotas.

Chordia (1996), inclusive, já evidenciou por meio da análise de 397 fundos mútuos estadunidenses com estratégias distintas, entre 1984 e 1993, que esses fundos elevaram a cobrança de suas taxas de saída, para minimizar a solicitação de resgates, além de mostrar que os fundos pesquisados mantêm maiores quantias em caixa quando o regaste das cotas é incerto.

Liang (1999), em pesquisa com 921 hedge funds estadunidenses, no período de 1994 a 1996, analisou a relação entre o desempenho desses fundos e a imposição de períodos de lock-up. Como resultados, evidenciou que maiores retornos estão ligados diretamente com o tempo de resgate, além da preservação da disponibilidade de caixa dos fundos.

Ljungqvist e Richardson (2003), em uma amostra de 73 fundos estadunidenses classificados como hedge funds, no período de 1981-2001, analisaram os impactos do fluxo de caixa sobre o desempenho das cotas desses fundos. Sob essa lógica, os resultados indicaram que, quanto menores forem as saídas de caixa e, quanto mais tardias essas saídas ocorrerem, maior será a rentabilidade das cotas.

Desse modo, constata-se que o estabelecimento de políticas que restringem a liquidez das cotas permite aos gestores de hedge funds explorar suas estratégias de investimento, pois, conforme destacam Agarwal et al. (2009, p. 2.223) "quanto mais longo for o período de lock-up, aviso de resgate, e período de resgate, maior é a liberdade do gestor para levar a cabo diferentes estratégias de investimento, sem se preocupar com a necessidade de liquidez do investidor".

Verifica-se que há consenso entre os estudos explicitados quanto ao efeito da imposição de períodos de lock-up sobre o saldo de disponibilidades, fato que pode afetar a rentabilidade das cotas dos fundos multimercados. Tal consenso permite realizar as seguintes inferências: i) períodos de lock-up são estabelecidos para que os fundos multimercados implementem suas estratégias, dentre elas, montar suas carteiras de investimentos; ii) períodos de lock-up acarretam menores investimentos no saldo de disponibilidades, ocasionando variações positivas; iii) na ausência de períodos de lock-up, a rentabilidade das cotas seria reduzida por meio da venda dos ativos financeiros para a cobertura de resgates. Tais argumentos corroboram, portanto, a hipótese $\left(\mathbf{H}_{1}\right)$ deste estudo:

H1: Períodos de lock-up apresentam efeito negativo sobre o saldo de disponibilidades mantido pelos fundos multimercados.

Aragon (2007) destaca que os hedge funds oferecem um ambiente ideal para pesquisar os dilemas de liquidez, visto que a imposição de períodos de lock-up permitem o melhor gerenciamento dos ativos, em função de contribuírem para a redução dos desembolsos. Nesse sentido, Malaquias, Malaquias, Souza e Mamede (2016) corroboram ao recomendarem que estudos futuros na catego- 
ria dos fundos multimercados explorem essa relação, visto que a imposição de restrições e a baixa liquidez das cotas podem conduzir à rentabilidade das cotas desses fundos.

\subsection{Variáveis que Afetam o Desempenho dos Fundos Multimercados}

Além de períodos de lock-up, outras variáveis têm sido empregadas na literatura para explicar o desempenho dos fundos multimercados como taxa de administração, taxa de performance e tamanho dos fundos (Amin \& Kat, 2003; Malaquias \& Eid, 2014; Pontes et al., 2015).

Damke, Eid e Rochman (2014) esclarecem que, no Brasil, a CVM prevê uma diferença formal entre a função do administrador e a do gestor, que parece não ocorrer com os hedge funds nos Estados Unidos. No Art. $78 \S 1^{\circ}$ da Instrução CVM nº 555 de 2014 (dispõe sobre a constituição, a administração, o funcionamento e a divulgação de informações dos fundos de investimento) verifica-se que os administradores correspondem "as pessoas jurídicas autorizadas pela CVM para o exercício profissional de administração de carteiras de valores mobiliários” Já no $\S 3^{\text {o }}$ do Art. 78 tem-se que o gestor trata-se da "pessoa natural ou jurídica credenciada como administradora de carteiras de valores mobiliários pela CVM" (INSTRUÇÃO CVM, 2014).

Aragon e Nanda (2012) explicam que a cobrança de taxas de administração se baseia no percentual de ativos que são mantidos e administrados pelos fundos. Interessante notar que a cobrança dessas taxas conduz os investidores a vislumbrarem carteiras de ativos com maior potencial de ganhos, o que justifica, portanto, a imposição dessas taxas. Apesar disso, alguns estudos têm evidenciado que as taxas de administração podem corroer a rentabilidade das cotas (Amin \& Kat, 2003; Rochman \& Ribeiro, 2003).

Assim como evidenciado por Malaquias e Eid (2014), Pontes et al. (2015) constataram um efeito negativo na cobrança de taxas de administração sobre o desempenho dos fundos brasileiros long and short. Os resultados destas pesquisas mostram que a rentabilidade auferida pelas cotas pode não ser transferida aos investidores, dado que o benefício gerado pela captação de renda pelos ativos pode ser consumido por meio da cobrança dessa taxa, o que reduz a rentabilidade bruta das cotas dos fundos multimercados.

Sobre a cobrança de taxas de performance, Varga (2001) cita que os gestores que conseguem superar os benchmarks do mercado são vistos como os profissionais que apresentam melhor desempenho. Berk e Green (2004) corroboram essa ideia, ao dizer que esse aspecto pode ser determinante na escolha de um investidor por um fundo de investimento, já que o desempenho das cotas de um fundo é interpretado como sinônimo de habilidade superior na seleção de ativos mais rentáveis.

Buraschi, Kosowski e Sritrakul (2014) dizem que a cobrança da taxa de performance ocorre se o gestor do fundo aumentar o patrimônio líquido do período atual em relação ao desempenho do período anterior. Assim, se os gestores conseguem superar o benchmark trazido pelo prospecto dos fundos e elevar a sua riqueza em relação ao período anterior, as cotas são acometidas pela cobrança da taxa de performance. Logo, a rentabilidade das cotas é influenciada positivamente pela cobrança de taxas de performance, mesmo que o seu recolhimento implique a redução dos valores que são transferidos para os cotistas. No estudo de Ackermann et al. (1999) a cobrança da taxa de performance apresentou efeito positivo sobre o desempenho das cotas de hedge funds estadunidenses, contudo, esse efeito não foi corroborado nas pesquisas de Malaquias e Eid (2014) e Pontes et al. (2015), no contexto dos fundos multimercados brasileiros.

No que refere à variável tamanho dos fundos, Ackermann et al. (1999) afirmam que essa característica é partilhada em trabalhos sobre fundos de investimento, em razão das economias de escala que os fundos conseguem obter pela quantidade de ativos sob a sua posse, fato que pode 
impactar na sua rentabilidade. Além disso, esse fator pode ser um indicativo de que um fundo faz a opção por estratégias de arbitragem, quando não dispõe de altas quantias de recursos.

Conforme esclarecem Bali, Gockan e Liang (2007), fundos com menor porte podem encerrar as suas operações em detrimento da falta de recursos para superar os gastos de suas operações. $\mathrm{O}$ tamanho dos fundos parece ser um aspecto bastante relevante para os hedge funds, em virtude de reputação: gestores que estão iniciando em suas carreiras, dispõem de incentivos apreciáveis para que aumentem a quantidade de ativos gerenciados (Aggarwal \& Jorion, 2010).

Chan, Faff, Gallagher e Looi (2009) em uma amostra formada a partir de dados de desempenho cedidos por gestores de fundos de ações australianos, no período de 1998 a 2001, constataram que fundos maiores têm o seu desempenho diminuído em função dos denominados custos de impacto de mercado e pela atividade de negociação dos gestores. Busse, Chordia, Jiang e Tang (2014) afirmam que no contexto dos fundos mútuos é consenso na literatura que ocorra a diminuição da rentabilidade em fundos maiores por causa dos custos de transação. Contudo, analisando uma amostra de 5.469 fundos mútuos estadunidenses, no período de 1980 a 2012, evidenciaram que fundos maiores incorreram em menores custos de transação frente aos fundos que gerenciam menores patrimônios. Tal explicação estaria ligada à manutenção de carteiras mais líquidas pelos fundos maiores, gerando, por conseguinte, um prêmio pelo risco menor, fato este oposto no ambiente dos fundos menores.

Bollen e Pool (2009) explicam que o tamanho do fundo pode ser mensurado por meio da média dos ativos registrados pelos fundos. Entretanto, verifica-se que algumas pesquisas realizadas no Brasil utilizaram, como proxy para a mensuração dessa característica, o valor do patrimônio líquido ao invés do seu ativo total (Castro \& Minardi, 2009; Malaquias \& Eid, 2014). A explicação pode decorrer do fato de que a divisão do patrimônio líquido pelo número de cotas do fundo representa o valor da cota adquirida por cada investidor, implicando, portanto, em uma medida mais adequada para a sua mensuração. Destarte, o tamanho de um fundo, medido pelo valor do patrimônio líquido, pode afetar a rentabilidade das cotas de fundos multimercados, uma vez que algumas estratégias nesta categoria de fundos podem estar associadas com o tamanho da operação, de modo que sejam implementadas de forma rentável.

\subsection{Características dos Fundos Multimercados Brasileiros}

Malaquias e Eid (2014) esclarecem que os hedge funds se caracterizam pela aplicação de estratégias distintas das tradicionais (investimento em ações, renda fixa, câmbio), abrangendo operações com alavancagem e derivativos, apesar de essas estratégias não garantirem que esses fundos obtenham desempenho superior. Para Schutt e Caldeira (2016), o desempenho dos hedge funds é determinado por, pelo menos, dois fatores: a rentabilidade dos ativos que compõem a carteira de investimentos e a performance obtida pelas estratégias praticadas.

Outros autores sustentam que um dos fatores fundamentais para elucidar o desempenho dos hedge funds é retratado pela cobrança da taxa de performance, já que elas incentivam o gestor a atingir as metas estabelecidas (Ackermann et al.,1999; Agarwal et al., 2009).

Malaquias e Eid (2013) afirmam que o tamanho do fundo representa o motivo para um desempenho superior das cotas de hedge funds, visto que fundos maiores possuem ganhos de escala e poder de barganha. Todavia, Aragon (2007) defende que o desempenho das cotas de hedge funds é ocasionado pela imposição de restrições, como períodos de lock-up e não somente à habilidade do gestor.

Gomes e Cresto (2010) explicam que, apesar da semelhança, nem todos os fundos multimercados podem ser classificados como hedge funds, visto que algumas instituições, no Brasil, utili- 
zam essa classificação para conseguir facilidade de regulamentação, já que ela é o mais possível abrangente.

Mellone (2006) destaca a existência de, no mínimo, duas grandes diferenças entre os fundos multimercados no Brasil e os hedge funds dos Estados Unidos. A primeira consiste na escolha do mercado, pois a maior parte dos fundos multimercados realiza operações com ativos negociados no mercado brasileiro. Já os hedge funds realizam negociações com todos os mercados, de acordo com a oportunidade existente. A segunda diferença é a regulamentação, visto que os fundos multimercados são regulados pela mesma legislação que os demais fundos de investimento existentes, trazida pela CVM, enquanto que, os hedge funds possuem flexibilidade no aspecto regulatório, sendo considerados, legalmente, veículos privados de investimentos.

Fonseca, Bressan, Iquiapaza e Guerra (2007) caracterizam os fundos multimercados como uma forma de investimento que se baseia em alguns fatores de risco, sem compromisso de concentração em um ativo exclusivo. Esses fundos utilizam estratégias de renda fixa, câmbio, ações entre outras. Dessa forma, por não se concentrarem em um único ativo, os fundos multimercados mantêm as carteiras diversificadas, ou seja, o risco total é reduzido já que todo o seu patrimônio estará aplicado em diversos ativos, pois, quanto maior for a diversificação, mais seguras se tornarão as carteiras (Fonseca et al., 2007).

Yoshinaga, Castro, Oda e Lucchesi (2009) ressaltam que, devido às diversas opções de investimento que um fundo multimercado possui, é transferida para o gestor a responsabilidade de alocar os recursos da forma que julgar mais rentável para os cotistas. Portanto, Schutt e Caldeira (2016) defendem que os fundos multimercados são os que possuem maior liberdade de gestão, mas apresentam maior risco, à medida que utilizam diversas estratégias de aplicação de recursos e que não existe nenhuma referência de mercado amplamente aceita à qual possam ser comparados.

\section{PROCEDIMENTOS METODOLÓGICOS}

Esta pesquisa, do ponto de vista de sua natureza, pode ser classificada, segundo Barros e Lehfeld (2007), como aplicada, uma vez que o objetivo consiste em analisar se a imposição de períodos de lock-up reduz o saldo de disponibilidades dos fundos multimercados brasileiros.

Do ponto de vista da forma de abordagem, trata-se de uma pesquisa quantitativa, pois de acordo com Barros e Lehfeld (2007), busca a confirmação das suposições mediante a utilização de dados estruturados e estatísticos.

Sob o ponto de vista dos objetivos, a presente pesquisa pode ser classificada, segundo Marconi e Lakatos (2011), como descritiva, dado que busca estabelecer uma relação entre períodos de lock-up e o saldo de disponibilidades, a fim de propiciar indícios de formas de obtenção de rentabilidade pelos fundos multimercados brasileiros.

Salienta-se que a categoria dos fundos multimercados brasileiros foi selecionada para esta pesquisa, devido ao desempenho que as cotas desses fundos apresentam aos seus investidores. No ano de 2007, por exemplo, esses fundos ofereceram quase $10 \%$ a mais de retorno em relação aos fundos de renda fixa (Gomes \& Cresto, 2010).

Para o desenvolvimento desta pesquisa, os dados que a compuseram se referem ao período de 2011 a 2015. Esse período foi escolhido, em função da disponibilidade dos dados no sistema Economática ${ }^{\circledR}$, contudo, salienta-se que ele é relevante para a análise, pois nesse período houve um crescimento significativo do patrimônio líquido dos fundos multimercados, chegando a ultrapassar dois trilhões de reais (Roquete et al., 2016).

Para a seleção dos fundos multimercados que compuseram a amostra final da pesquisa, alguns critérios foram adotados. Primeiramente, a partir dos 2.441 fundos disponibilizados no sistema Economática ${ }^{\circledR}$, optou-se por aqueles que detivessem maiores valores de patrimônio líquido 
(mediana acima de $\mathrm{R} \$ 31.229 .688,69$ ) e períodos de lock-up iguais ou superiores a 30 dias, o que totalizou 79 fundos multimercados. A escolha de períodos de lock-up superiores a 30 dias deu-se em razão de se poder comparar os resultados considerando fundos grandes que praticam algum período de restrição de resgate aos cotistas. Com isso, fundos que não possuem períodos de lock-up não fazem parte da amostra. Com esta decisão, a análise de potenciais efeitos de maiores períodos de lock-up no saldo de disponibilidades passa a ser feito apenas entre aqueles fundos que já possuem o período de lock-up, e ainda assim existe considerável variação entre estes fundos (conforme mostrado a seguir, os períodos variam de 30 a 180 dias nos fundos da amostra). Na sequência, coletaram-se manualmente no site da CVM (n.d.), os dados referentes ao saldo de disponibilidades mantido pelos 79 fundos multimercados. Desse modo, obtiveram-se 60 observações mensais sobre o saldo de disponibilidades e períodos de lock-up de cada fundo, totalizando assim, 4.740 observações no período de 2011 a 2015.

Para a análise dos dados, empregou-se a estatística descritiva, com a finalidade de expor a composição da variável períodos de lock-up e saldo de disponibilidades. Depois, foi empregada a análise de correlação para verificar se houve associação entre a imposição de períodos de lock-up e o saldo de disponibilidades mantido pelos fundos multimercados. Segundo Martins (2001, p. 287), “[...] a possível existência de relação entre variáveis orienta análises, conclusões e evidenciação de achados da investigação".

Ademais, empregou-se a análise de regressão para dados em painel, a fim de verificar o efeito da imposição de períodos de lock-up sobre o saldo de disponibilidades mantido pelos fundos multimercados brasileiros. A análise de regressão para dados em painel é uma técnica que visa a identificar os impactos que uma variável exerce sobre outra, ou seja, se a variação de uma pode influenciar a variação da outra (Hoffmann, 2015). Para o emprego desta técnica, utilizou-se a equação 1, apresentada a seguir:

Disponibilidadesit $=\beta_{0}+\beta_{1}$ Lock-up $p_{i t}+\beta_{2}$ Subcategoriait $+\beta_{3}$ Tadmit $+\beta_{4}$ Tpfmit $_{i t}+\beta_{5}$ Tamit $_{i}+\varepsilon$ (eq. 1)

Na Tabela 1, a seguir, são apresentadas as especificações das variáveis usadas na equação 1, tendo em vista o objetivo geral da presente pesquisa:

Tabela 1: Especificações das variáveis utilizadas e hipótese da pesquisa

\begin{tabular}{|c|c|c|c|c|}
\hline Variável & $\begin{array}{c}\text { Sinal } \\
\text { esperado }\end{array}$ & Significado & Referências & Fonte \\
\hline Disponibilidades & $\begin{array}{c}\text { Variável } \\
\text { dependente }\end{array}$ & $\begin{array}{c}\text { Saldo de } \\
\text { disponibilidades em } \\
\text { valores monetários } \\
\text { mantido pelos } \\
\text { fundos ao final de } \\
\text { cada mês. } \\
\text { Logaritmo natural } \\
+1 \text {. } \\
\end{array}$ & $\begin{array}{l}\text { Chordia (1996); Liang } \\
\text { (1999); Ackermann et al. } \\
\text { (1999); Sadka (2010); }\end{array}$ & CVM \\
\hline Lock-up & $+/-$ & $\begin{array}{l}\text { Quantidade de dias } \\
\text { entre a solicitação de } \\
\text { resgate pelo } \\
\text { investidor e o seu } \\
\text { efetivo recebimento. }\end{array}$ & $\begin{array}{c}\text { Aragon (2007); Agarwal et } \\
\text { al. (2009); Malaquias \& } \\
\text { Mamede (2015); Pontes et } \\
\text { al., (2015). }\end{array}$ & Economática ${ }^{\circledR}$ \\
\hline Subcategoria & $+/-$ & $\begin{array}{c}\text { Variável dummy: } 1= \\
\text { fundos } \\
\text { multimercados } \\
\text { Estratégia Livre; } 0=\end{array}$ & $\begin{array}{l}\text { Fonseca et al., (2007); } \\
\text { Yoshinaga et al., (2009); } \\
\text { Gomes \& Cresto (2010). }\end{array}$ & Economática ${ }^{\circledR}$ \\
\hline
\end{tabular}




\begin{tabular}{c|c|c|c|c}
\hline & & $\begin{array}{c}\text { pertence as outras } \\
\text { subcategorias. }\end{array}$ & & \\
\hline Taxa de Administração & - & $\begin{array}{c}\text { Taxa máxima de } \\
\text { administração } \\
\text { cobrada pelo fundo } \\
\text { anualmente. }\end{array}$ & $\begin{array}{c}\text { Amin \& Kat (2003); } \\
\text { Rochman \& Ribeiro } \\
(2003) ; \text { Malaquias \& Eid } \\
(2014) .\end{array}$ & Economática ${ }^{\circledR}$ \\
\hline Taxa de Performance & + & $\begin{array}{c}\text { Variável dummy: } 1= \\
\text { cobra taxa de } \\
\text { performance; } 0=\text { não } \\
\text { cobra taxa de } \\
\text { performance. }\end{array}$ & $\begin{array}{c}\text { Ackermann } \text { et al. (1999); } \\
\text { Berk \& Green (2004); } \\
\text { Buraschi } \text { et al. (2014). }\end{array}$ & Economática ${ }^{\circledR}$ \\
\hline Tamanho do Fundo & $+/-$ & $\begin{array}{c}\text { Logaritmo natural da } \\
\text { média do patrimônio } \\
\text { líquido do fundo no } \\
\text { período. }\end{array}$ & $\begin{array}{c}\text { Ackermann } \text { et al. (1999); } \\
\text { Busse } \text { et al. (2014); Castro } \\
\text { \& Minardi (2009). }\end{array}$ & Economática ${ }^{\circledR}$ \\
\hline
\end{tabular}

H1: Períodos de lock-up apresentam efeito sobre o saldo de disponibilidades mantido pelos fundos multimercados.

Fonte: elaborada pelos autores.

Na equação 1, a subcategoria refere-se à uma variável dummy, atribuída à subcategoria Estratégia livre foi utilizada no modelo como variável de controle, a fim de captar o efeito da subcategoria sobre o saldo de disponibilidades mantido pelos fundos multimercados, bem como para evitar problemas de multicolinearidade, caso fossem atribuídas variáveis dummies para as demais subcategorias. Sua inclusão baseou-se na consulta a pesquisas anteriores, como Fonseca et al., (2007), Yoshinaga et al., (2009) e Gomes e Cresto (2010). Assim, os fundos multimercados Estratégia Livre foram escolhidos, por serem a subcategoria com maior número de observações na amostra pesquisada (1.920 observações). Por tratar-se de uma variável de controle, não foi especificado um sinal para o seu potencial efeito no saldo de disponibilidades. Ademais, o modelo quantitativo apresentado na equação 1 conta também com outras três variáveis de controle inseridas com base em estudos anteriores (Malaquias \& Eid, 2014; Borges \& Martelanc, 2015), sendo elas: taxa de administração (mensurada pela taxa máxima de administração cobrada pelo fundo anualmente); taxa de performance (dummy que indica se o fundo possui ou não possui taxa de performance); tamanho (representado pelo logaritmo natural da média do patrimônio líquido do fundo no período).

Para a escolha do tipo de painel, em virtude das características das variáveis empregadas, utilizou-se apenas o teste Lagrange Multiplier (LM) de Breusch e Pagan, que compara o emprego do modelo pooled e o modelo de efeitos aleatórios. Posteriormente, os outputs do modelo apresentado na equação 1 foram analisados em relação à ocorrência de problemas de multicolinearidade e heterocedasticidade, por meio do teste de Fator de Inflação da Variância (FIV) e do teste de Breusch e Pagan, respectivamente, de modo que tais problemas fossem corrigidos. Para o tratamento da heterocedasticidade, empregou-se a correção robusta de White, conforme sugerem Gujarati e Porter (2011).

Como forma de análise de robustez dos resultados, aplicou-se o procedimento de winsorize na variável dependente, bem como foi realizado o cálculo de seu logaritmo natural, o que permite eliminar elevadas diferenças nos saldos de disponibilidades registrados pelos fundos da amostra. No que se refere ao procedimento winsorize, adotou-se o valor de 2,5\% nos maiores e menores valores da variável dependente (que corresponde aos 118 menores valores e aos 118 maiores valores, sendo substituídos pelo valor subsequente na posição 119). O valor de $2,5 \%$ foi escolhido por proporcionar o tratamento de uma pequena parte do banco de dados, mas que já possibilita a eliminação de extreme outliers.

\section{ANÁLISE DE RESULTADO}


A Tabela 2 resume os resultados encontrados após o uso da estatística descritiva para as variáveis períodos de lock-up e disponibilidades, para as 4.740 observações analisadas nesta pesquisa:

Tabela 2: Estatística descritiva das variáveis analisadas

\begin{tabular}{|c|c|c|c|c|c|}
\hline Variável & $\begin{array}{c}\text { № de observa- } \\
\text { ções }\end{array}$ & Média & Desvio Padrão & Mínimo & Máximo \\
\hline Disponibilidades (R\$) & 4.740 & $130.281,80$ & $2.231 .480,00$ & $-588.424,40$ & $96.373 .976,02$ \\
\hline Disponibilidades (R\$) (w) & 4.740 & $25.734,08$ & $94.024,580$ & 0,000 & $545.520,900$ \\
\hline Ln de Disponibilidades (R\$) (w) & 4.740 & 7,105 & 3,390 & 0,000 & 13,210 \\
\hline Lock-up (dias) & 4.740 & 46,400 & 31,240 & 30,000 & 180,000 \\
\hline Subcategoria & 4.740 & 0,405 & 0,491 & 0,000 & 1,000 \\
\hline Tadm & 4.740 & 1,529 & 1,062 & 0,000 & 4,000 \\
\hline Tpfm & 4.740 & 0,456 & 0,498 & 0,000 & 1,000 \\
\hline Tamanho & 4.740 & 18,794 & 1,076 & 17,318 & 21,473 \\
\hline
\end{tabular}

Fonte: resultados da pesquisa.

Tendo em vista os dados da Tabela 2, verifica-se que a média do saldo de disponibilidades dos fundos multimercados analisados foi de $\mathrm{R} \$ 130.281,80$, e dos períodos de lock-up de aproximadamente 46 dias. Comparando com a pesquisa de Aragon (2007) e Agarwal et al. (2009), em que a média dos períodos de lock-up foi de um ano, pode-se inferir que o período de lock-up encontrado nesta pesquisa foi relativamente menor, uma vez que o maior período de lock-up foi de 180 dias. Com base na elevada dispersão apresentada pela variável disponibilidades, realizou-se um procedimento adicional de winsorize (a 2,5\%), com o objetivo de eliminar potenciais vieses decorrentes de valores extremos. A segunda linha da tabela representa a estatística descritiva da variável disponibilidades após o procedimento de winsorize.

Adicionalmente, considerando que ainda poderia haver algum viés de diferença de escala, foi utilizada a transformação da variável disponibilidades (após o procedimento de winsorize) por meio do cálculo do seu logaritmo natural (neste caso, fez-se o logaritmo natural +1 , em função de algumas observações serem iguais a zero). Constata-se também que 40,5\% das observações é proveniente de fundos da categoria Multimercados Estratégia Livre e que aproximadamente $46 \%$ dos fundos da amostra cobram taxa de performance. De forma complementar, a Tabela 3 evidencia o comportamento da média e mediana do saldo da variável dependente (saldo de disponibilidades) ao longo do período da pesquisa.

Tabela 3: Média do saldo de disponibilidades ao longo do período da amostra

\begin{tabular}{lcc}
\hline Ano & No de observações & Média \\
\hline 2011 & 948 & $26.746,60$ \\
2012 & 948 & $18.374,30$ \\
2013 & 948 & $31.742,30$
\end{tabular}


Notas: os resultados desta tabela referem-se ao saldo de disponibilidades, em $\mathrm{R} \$$, após o procedimento winsorize. Fonte: resultados da pesquisa.

A partir da base de dados desta pesquisa, foi possível identificar que o saldo negativo em disponibilidades de $\mathrm{R} \$ 588.424,40$ esteve relacionado com o fundo que fixou o máximo de 180 dias de períodos de lock-up. Já o fundo que apresentou o maior saldo de disponibilidades, no valor de $\mathrm{R}$ \$96.373.976,02, detinha em sua política 31 dias de períodos de lock-up. Na Tabela 4, esses argumentos são novamente analisados, levando-se em consideração o teste de correlação de Pearson entre as variáveis períodos de lock-up e disponibilidades:

Tabela 4: Teste de correlação de Pearson

\begin{tabular}{clcccccc}
\hline Variáveis & & $\mathbf{A}$ & $\mathbf{B}$ & $\mathbf{C}$ & $\mathbf{D}$ & $\mathbf{E}$ & $\mathbf{F}$ \\
\hline A & Disponibilidades (R $\$)$ & 1,000 & & & & & \\
B & Lock-up (dias) & $-0,022$ & 1,000 & & & & \\
C & Subcategoria & 0,052 & 0,112 & 1,000 & & & \\
D & Tadm & 0,050 & $-0,134$ & $-0,200$ & 1,000 & & \\
E & Tpfm & 0,032 & 0,065 & $-0,134$ & 0,284 & 1,000 & \\
F & Tamanho & $-0,026$ & $-0,060$ & $-0,113$ & 0,126 & 0,187 & 1,000 \\
\hline
\end{tabular}

Nota. A correlação entre as variáveis Lock-up e o logaritmo natural de Disponibilidades foi equivalente (igual a -0,0503).

Fonte: resultados da pesquisa.

De acordo com o teste de correlação de Pearson, expresso pelo coeficiente de correlação de 0,0222 na Tabela 4, pode-se afirmar no nível nominal de significância de 5\%, que não houve correlação entre a imposição de períodos de lock-up e o saldo de disponibilidades mantido pelos fundos multimercados desta pesquisa. Portanto, com base na análise bivariada, quando o período de lockup aumentou ou diminuiu, não significa dizer que o saldo de disponibilidades aumentou ou diminuiu na mesma proporção. Essa constatação reforça os argumentos anteriores de que, no Brasil, essa política necessariamente não tem sido utilizada pelos fundos multimercados para obterem maior rentabilidade, não corroborando dessa forma Liang (1999), Ackermann et al. (1999) e Ljungqvist e Richardson (2003), quanto ao efeito dos períodos de lock-up sobre o saldo de disponibilidades.

Para o teste da hipótese definida nesta pesquisa, na sequência, são demonstrados na Tabela 5 os testes precedentes ao modelo para dados em painel:

Tabela 5: Teste FIV, Heterocedasticidade e LM

\begin{tabular}{cc|c|c}
\hline Fator de Inflação de Variância (FIV) & $\begin{array}{c}\text { Heterocedasticidade } \\
\text { de Breusch e Pagan }\end{array}$ & $\begin{array}{c}\text { Lagrange Multiplier } \\
\text { de Breusch-Pagan }\end{array}$ \\
\hline Variáveis & Resultados & & Chi $^{2}: 829,78$ \\
Tadm & 1,150 & Chi $^{2}: 12,492,05$ & \\
Tpfm & 1,140 & & Valor-p: $0,000^{* * *}$ \\
Subcategoria & 1,060 & Valor-p: $0,000^{* * *}$ & \\
Tamanho & 1,050 & & \\
Lock-up (dias) & 1,040 & & \\
\hline
\end{tabular}

Nota. Nível nominal de significância estatística: ${ }^{*}$ valor- $p<10 \%$; ${ }^{* *}$ valor-p $<5 \%$; ${ }^{* * *}$ valor-p $<1 \%$.

Fonte: resultados da pesquisa. 
A partir dos resultados expressos na Tabela 5, verifica-se que as variáveis independentes elencadas para o modelo não apresentam multicolinearidade, ou seja, como o resultado do fator de inflação de variância foi menor que dez, cada uma dessas variáveis contribui para a explicação da variável dependente (Gujarati \& Porter, 2011).

Tendo em vista o teste de Breusch e Pagan, em nível nominal de significância de 5\%, pode-se afirmar que os dados da pesquisa são heterocedásticos. Segundo Gujarati e Porter (2011), uma das explicações para esse resultado pode ser atribuída a presença dos extreme outliers nos resíduos da regressão, ou seja, números com valores discrepantes, o que pode ser conferido à elevada variabilidade apresentada pela variável saldo de disponibilidades dos fundos multimercados pesquisados. Nesse sentido, algumas alternativas são indicadas, como a correção robusta de White para heterocedasticidade (Gujarati \& Porter, 2011).

Quanto à escolha entre o modelo pooled e o modelo de efeitos aleatórios para dados em painel, em nível nominal de significância de 5\%, o teste Lagrange Multiplier de Breusch-Pagan trouxe que a especificação adequada do modelo para dados em painel corresponde ao modelo de efeitos aleatórios (valor-p $=0,000$ ). Como a influência do tempo é relevante para os dados da pesquisa, esta pode ser a provável explicação para a especificação obtida no teste.

Na Tabela 6, têm-se os resultados para a variável dependente saldo de disponibilidades, estimados sob a perspectiva do modelo de efeitos aleatórios com correção robusta de White antes e após o tratamento dos extreme outliers.

Tabela 6: Análise do potencial efeito do período de Lock-up no saldo de disponibilidades

\begin{tabular}{lcc}
\hline Variável Dependente & $\begin{array}{c}\text { Saldo de Disponibilidades } \\
\text { (sem transformação) }\end{array}$ & $\begin{array}{c}\text { LN do Saldo de Dispo- } \\
\text { nibilidades (Winsorize) }\end{array}$ \\
\hline Variáveis Independentes & Coeficientes & Coeficientes \\
Lock-up (dias) & $-1.905,2$ & $-0,011$ \\
Subcategoria & $304.299,9$ & 0,898 \\
Tadm & $113.818,8$ & $0,447 * *$ \\
Tpfm & $149.047,5$ & $-0,900$ \\
Tamanho & $-69.442,4$ & 0,011 \\
Constante & $1.158 .520,0$ & 6,761 \\
\hline$N^{o}$ de observações & 4.740 & 4.740 \\
Quantidade de fundos & 79 & 79 \\
\hline $\mathrm{R}^{2}$ _overall & 0,009 & 0,054 \\
$\mathrm{R}^{2}$ _between & 0,087 & 0,065 \\
$\mathrm{R}^{2}$ _within & 0,000 & 0,000 \\
Chi ${ }^{2}$ & 3,050 & 12,690 \\
\hline
\end{tabular}

Nota. Nível nominal de significância estatística: ${ }^{*}$ valor- $p<10 \%$; ${ }^{* *}$ valor-p $<5 \%$; ${ }^{* * *}$ valor-p $<1 \%$.

Variável dependente: disponível mensal ou logaritmo natural do saldo de disponibilidades dos fundos multimercados analisados; Lock-up: quantidade de dias que o investidor deve permanecer com suas aplicações em uma subcategoria de fundos multimercados; Dummy Subcategoria: 1 se pertence a subcategoria Multimercados Estratégia Livre, 0 se pertence às demais categorias; taxa de administração: mensurada pela taxa máxima de administração cobrada pelo fundo anualmente; taxa de performance: dummy que indica se o fundo possui ou não possui taxa de performance; tamanho: representado pelo logaritmo natural da média do patrimônio líquido do fundo no período.

Fonte: resultados da pesquisa.

Os resultados evidenciados na Tabela 6 mostram que a hipótese definida nesta pesquisa não foi sustentada, ou seja, a imposição de períodos de lock-up não necessariamente apresentou um efeito (e estatisticamente significativo) sobre o saldo de disponibilidades mantido pelos fundos multimercados brasileiros. No contexto dos fundos mútuos, Chordia (1996) evidenciou que essa categoria de fundos costuma manter maiores quantias em caixa, quando o regaste das cotas é in- 
certo. Entretanto, é oportuno também comentar que altos investimentos em saldo de caixa podem proporcionar aumento nos custos de oportunidade. Os resultados indicam ainda que a subcategoria Multimercados Estratégia Livre pode apresentar maiores saldos em disponibilidades, mas a significância não foi persistente com a correção robusta de White para a amostra geral. A ausência de efeito significativo da variável tamanho talvez esteja relacionada com o critério de seleção da amostra utilizada neste estudo, no qual são considerados fundos maiores.

Por fim, com o tratamento dos extreme outliers (neste caso, foi utilizada como variável dependente o logaritmo natural do saldo de disponibilidades após o procedimento de winsorize), verifica-se que o efeito da imposição de períodos de lock-up sobre o saldo de disponibilidades manteve-se como não significativo, corroborando os resultados antes do tratamento dos extreme outliers. Quanto às variáveis de controle, o efeito delas sobre o saldo de disponibilidades também não foi significativo, exceto para a variável Taxa de Administração, o que sugere que fundos que cobram maiores taxas de administração tendem a apresentar maiores saldos em disponibilidades. De maneira geral, para a amostra como um todo, os resultados expressos reiteram a rejeição da hipótese principal definida para esta pesquisa.

Em uma análise adicional para fins de robustez considerando interação entre as variáveis dummy para categoria e período de lock-up (transformada em Ln), observou-se um valor positivo para o coeficiente desta variável de interação (e estatisticamente significativo a 1\%), indicando que, na subcategoria de Multimercados Estratégia Livre, maiores períodos de lock-up podem estar associados com maiores níveis de saldo mantido em disponibilidades. Embora o efeito seja significativo, ainda não se mostrou em linha com os argumentos desde estudo de que maiores períodos de lock-up poderiam estar associados com menores níveis de saldo de disponibilidades.

\section{CONCLUSÃO}

Este trabalho foi desenvolvido com o objetivo de analisar se a imposição de períodos de lock-up reduziu o saldo de disponibilidades dos fundos multimercados brasileiros, no período de 2011 a 2015. A categoria de fundos multimercados foi escolhida, por se destacar no setor de fundos de investimento brasileiro nos últimos anos.

Após a análise de 4.740 observações, referentes a 79 fundos multimercados no período selecionado, os resultados obtidos para a mostra geral mostram que a imposição de períodos de lockup não necessariamente provocou um efeito negativo sobre o saldo de disponibilidades dos fundos. Uma explicação contundente com os resultados encontrados pode estar pautada na estatística descritiva. Como evidenciado, a média de dias estabelecidos para a imposição de períodos de lockup, foi de 46 dias. Em estudos realizados em outros países (Aragon, 2007; Agarwal et al., 2009), o prazo médio de lock-up foi de um ano, assim, o período encontrado nesta pesquisa pode ser considerado curto, o que pode afetar a liberdade dos gestores na gestão das carteiras de investimento dos fundos amostrados.

Uma segunda explicação apontada na análise dos resultados, se dá com base em Chordia (1996), dado que fundos que não operam com períodos de lock-up, manteriam maiores valores em caixa, já que, em qualquer momento, teriam que cobrir resgates imprevisíveis. Além disso, no Brasil, parece não existir o prêmio de lock-up na categoria dos fundos multimercados (pelo menos na sub-categoria long and short), o que justificaria uma potencial diminuição do saldo de disponibilidades na presença dessa política. Isso está em consonância com os achados de Pontes et al. (2015).

Ao analisar um potencial efeito de interação entre as variáveis de período de lock-up e a dummy para sub-categoria dos fundos, observou-se um efeito inverso ao esperado, indicando que na categoria de Multimercados Estratégia Livre, maiores períodos de lock-up podem estar associados com maiores saldos de disponibilidades mantidos pelos fundos. Esses resultados estão em coerência com as considerações feitas anteriormente relativas ao prazo de lock-up dos fundos da 
amostra ser menor em comparação com os prazos de lock-up de fundos de amostras de outros estudos, o que poderia demandar saldo de disponibilidades mesmo no contexto de restrições de resgates.

Como limitação, citam-se os critérios empregados na seleção dos fundos multimercados da presente pesquisa, pois, dado que os fundos escolhidos tiveram maiores valores de patrimônio líquido e períodos de lock-up iguais ou superiores a 30 dias, tais fundos podem apresentar melhor performance, o que pode refletir nas quantias mantidas em caixa. Ademais, a inclusão de outras variáveis explicativas nos modelos econométricos poderia, talvez, conduzir a resultados distintos dos evidenciados neste estudo. Por exemplo, poderia ser realizada a ponderação do saldo de disponibilidades pelo patrimônio do fundo no período imediatamente anterior (os fundos selecionados para este estudo já fazem parte de uma sub-amostra de fundos com maior valor de patrimônio líquido).

Esta pesquisa contribui para o avanço de estudos relacionados ao contexto dos fundos multimercados no Brasil, já que propicia a utilização de novas variáveis que ajudam a explicar o modo pelo qual essa categoria de fundos pode obter a rentabilidade de suas cotas, o que abre espaço para a realização de novas pesquisas sobre o tema.

\section{REFERÊNCIAS}

Ackermann, C., McEnally, R., \& Ravenscraft, D. (1999). The performance of hedge funds: risk, return, and incentives. The Journal of Finance, 54(3), 833-874.

Agarwal, V., Daniel, N. D., \& Naik, N. Y. (2009). Role of managerial incentives and discretion in hedge fund performance. The Journal of Finance, 64(5), 2221-2256.

Aggarwal, R. K., \& Jorion, P. (2010). The performance of emerging hedge funds and managers. Journal of Financial Economics, 96(2), 238-256.

Alonso, E. J. M. (2008). Hedge funds, riesgo bancário y disciplina de mercado. Boletín Económico de Ice, 2939(1), 51-59.

Amin, G. S., \&; Kat, H. M. (2003). Hedge fund performance 1990-2000: Do the "money machines" really add value? Journal of Financial and Quantitative Analysis, 38(2), 251-274.

Aragon, G. O. (2007). Share restrictions and asset pricing: evidence from the hedge fund industry. Journal of Financial Economics, 83(1), 33-58.

Aragon, G. O., \& Nanda, V. (2012). Tournament behavior in hedge funds: high-water marks, fund liquidation, and managerial stake. The Review of Financial Studies, 25(3), 937-974.

Associação Brasileira das Entidades dos Mercados Financeiro e de Capitais (n.d.). Com boom dos multimercados, ANBIMA intensifica supervisão de suitability. Recuperado em 20 de maio, 2018, de http://www.anbima.com.br/pt_br/imprensa/com-boom-dos-multimercados-anbimaintensifica-supervisao-de-suitability.htm.

Bali, T. G., Gokcan, S., \& Liang, B. (2007). Value at risk and the cross-section of hedge fund returns. Journal of Banking \& Finance, 31(4), 1135-1166.

Barros, A. J. S., \& Lehfeld, N. A. S. (2007). Fundamentos de Metodologia Científica (3a ed.). São Paulo: Pearson Prentice Hall. 
Berk, J., \& Green, R. (2004). Mutual fund flows and performance in rational markets. Journal of Political Economy, 112(6), 1269-1295.

Bollen, N. P. B., \& Pool, V. K. (2009). Do hedge fund managers misreport returns? Evidence from the pooled distribution. The Journal of Finance, 64(5), 2257-2288.

Borges, E. C., \& Martelanc, R. (2015). Sorte ou habilidade: uma avaliação dos fundos de investimento no Brasil. Revista de Administração 50(2), 196-207.

Buraschi, A., Kosowski, R., \& Sritrakul, W. (2014). Incentives and endogenous risk taking: a structural view on hedge fund alphas. Journal of Finance, 69(6), 2819-2870.

Busse, J. A, Chordia, T., Jiang, L., \& Tang, Y. (2014). How does size affect mutual fund performance? Evidence from mutual fund trades [Working Paper]. Research Collection Lee Kong Chian School Of Business. Recuperado de http://www.ckgsb.edu.cn/uploads/201409/paper\%20of\%20Prof.\%20Tarun\%20Chordia.pdf

Caldeira, J. F, Moura, G. V., Santos, A. A. P., \& Tessari, C. (2014). Seleção de carteiras com modelos fatoriais heterocedásticos: aplicação para fundos de fundos multimercados. Revista de Administração Mackenzie 15(2), 127-161.

Castro, B. R., \& Minardi, A. M. A. F. (2009). Comparação do Desempenho dos Fundos de Ações Ativos e Passivos. Revista Brasileira de Finanças, 7(2), 143-161.

Chan, H. W. H., Faff, R. W., Gallagher, D. R., \& Looi, A. (2009). Fund size, transaction costs and performance: Size matters! Australian Journal of Management, 34(1), 73-96.

Chordia, T. (1996). The structure of mutual fund charges. Journal of Financial Economics 41(1), 3-9.

Comissão de Valores Mobiliários. (n.d.). Recuperado em 14 setembro, 2017, de http://www.cvm.gov.br/menu/regulados/fundos/consultas/fundos/fundos.html

Damke, B. R., Eid, W., Jr, \& Rochman, R. R. (2014) Which are the investment fund managers in Brazil behavioral investing biases and their characteristics? In: Anais I Encontro Brasileiro de Economia e Finanças Comportamentais. São Paulo/SP.

Economática. (n.d.). Banco de dados. Recuperado em 6 janeiro, 2017, de http://www.economatica.com.br/index_fla.htm.

Eid, W., Jr., \& Rochman, R. R. (2014). Perspectivas para a indústria brasileira de fundos. Anuário ANBIMA 2014. Recuperado em 20 de maio de 2015, de http://www.anbima.com.br/anuariodefundos/2014/pt/A_Industria_de_fundos/Investimento_ no_Brasil/default.aspx.

Fonseca, N. F., Bressan, A. A., Iquiapaza, R. A., \& Guerra, J. P. (2007). Análise do desempenho recente de fundos de investimento no Brasil. Contabilidade Vista \& Revista, 18(1), 95-116.

Fonseca, S. C., \& Malaquias, R. F. (2012). O efeito smart Money no segmento de fundos multimercados. Revista de Gestão, Finanças e Contabilidade, 2(3), 3-16. 
Gomes, F. A. R., \& Cresto, V. (2010). Avaliação do desempenho dos fundos Long And Short no Brasil. Revista Brasileira de Finanças, 8(4), 505-529.

Gujarati, D., \& Porter, D. C. (2011). Econometria básica (5a ed.). Porto Alegre: McGraw Hill.

Hoffmann, R. (2015). Análise de regressão: uma introdução à econometria. Piracicaba: ESALQ/USP.

Hong, X. (2014). The dynamics of hedge fund share restrictions. Journal of Banking \& Finance, 49(1), 82-99.

Instrução CVM no 555, de 17 de dezembro de 2014. (2014). Dispõe sobre a constituição, a administração, o funcionamento e a divulgação de informações dos fundos de investimento. Recuperado de http://www.cvm.gov.br

Jordão, G. A., \& Moura, M. L. (2011). Performance analysis of brazilian hedge funds. Journal of Multinational Financial Management, 21(3), 165-176.

Liang, B. (1999). On the performance of hedge funds. Financial Analysts Journal, 55(4), 72-85.

Ljungqvist, A., \& Richardson, M. (2003). The cash flow, return and risk characteristics of private equity. [Working Paper n⿳0 03-001] New York University, New York, United States of America.

Malaquias, F. F. O., Malaquias, R. F., Souza, F. E., Mamede, S. P. N., \& Oliveira, A. C. L. (2016). O efeito smart money em períodos de crise financeira. Revista Ambiente Contábil, 8(1), 323-342.

Malaquias, R. F., \& Eid W., Jr. (2013). Eficiência de Mercado e Desempenho de Fundos Multimercados. Revista Brasileira de Finanças, 11(1), 119-142.

Malaquias, R. F., \& Eid W., Jr. (2014). Fundos multimercados: desempenho, determinantes do desempenho e efeito moderador. Revista de Administração Mackenzie, 15(4), 135-163.

Malaquias, R. F., \& Mamede, S. P. N. (2015). Efeito Calendário e Finanças Comportamentais no Segmento de Fundos Multimercados. RAC - Revista de Administração Contemporânea, 19, 98116 [Edição Especial].

Marconi, M. A., \& Lakatos, E. M. (2011). Técnicas de Pesquisa: planejamento e execução de pesquisas, amostragens e técnicas de pesquisa, elaboração, análise e interpretação de dados (7a ed.). São Paulo: Atlas.

Martins, G. A. (2001). Estatística geral e aplicada (2a ed.) São Paulo: Atlas.

Mellone, G. Jr. (2006). Especial Bancos: Fundos Multimercados. Revista GV executivo 5(3), 58-62.

Nanda, V., Narayanan, M. P., \& Warther, V. A. (2000). Liquidity, investment ability, and mutual fund structure. Journal of Financial Economics, 57(3), 417-443.

Pontes, G. A., Rogers, P., \& Malaquias, R. F. (2015). Os fundos Long and Short entregam o prêmio de lock-up? Evidências empíricas no Brasil. Revista Contabilidade Vista e Revista, 26(3), 106-123. 
Rochman, R. R., \& Eid, W, Jr. (2007). Fundos de investimento ativos e passivos no Brasil: comparando e determinando os seus desempenhos. In: Anais VII Encontro Brasileiro de Finanças. São Paulo/SP.

Rochman, R. R., \& Ribeiro, M. P. (2003, julho). A Relação entre a estrutura, conduta e desempenho da Indústria de Fundos de Investimento: um estudo de painel. Anais do Encontro Brasileiro de Finanças, São Paulo, SP, Brasil, 3.

Roquete, R. M., Maranho, F. S., Klotzle, M. C., \& Pinto, A. C. F. (2016). O Problema de Agência Aplicado aos Fundos de Investimento Multimercados. Revista de Finanças Aplicadas, 7(1), 1-21.

Sadka, R. (2010). Liquidity risk and the cross-section of hedge-fund returns. Journal of Financial Economics, 98(1), 4-71.

Schutt, I. G., Caldeira, J. F. (2016). Análise de Estilo Dinâmica de Fundos Multimercados: Aplicação para o Mercado Brasileiro. Revista Análise Econômica, 34(65), 101-129.

Varga, G. (2001). Índice de Sharpe e outros indicadores de performance aplicados a fundos de ações brasileiros. Revista de Administração Contemporânea, 5(3), 215-245.

Yoshinaga, C. E., Castro, F. H. F., Jr., ODA, A. L., \& Lucchesi, E. P. (2009). Análise de Estilo em Fundos Multimercados com e sem Alavancagem no Brasil. REGES - Revista Eletrônica de Gestão, 2(1), 9-21. 